\title{
La Sucesión en las Empresas Familiares: Una Discusión a partir de la Identidad Organizacional ${ }^{1}$
}

\author{
Mariana Lima Bandeira ${ }^{2}$ \\ Fernando López Parra ${ }^{3}$ \\ Marcelo Reinoso Navarro 4 \\ Universidad Andina Simón Bolívar
}

\section{RESUMEN}

Este estudio propone que la sucesión en una empresa familiar es, también, una cuestión de identidad organizacional. Nuestro argumento se construye a partir de la idea de que la sucesión en la empresa familiar es posible cuando hay un fuerte proceso de identificación con la empresa que no necesariamente está vinculado unilateralmente a la historia de la empresa familiar y a los lazos familiares directos, pero puede existir cuando las historias personales establecen un proceso de identificación con las historias de la familia. En este panorama, a partir de una revisión de la literatura sobre los procesos sucesorios en el ámbito de las empresas familiares y de la identidad organizacional, se construyen algunas reflexiones que podrían generar futuras investigaciones. Es un estudio que no se inscribe en la línea de estudios funcionalistas, toda vez que invita a una reflexión desde dinámicas intersubjetivas.

Palabras clave: Identidad Organizacional. Empresas Familiars. Procesos Sucesorios

\begin{abstract}
This study argues that succession in a family business is also a matter of organizational identity. Our argument is based on the idea that succession in the family business is possible when there is a strong process of identification with the business, which is not necessarily unilaterally linked to the history of the family business and direct family ties, but may exist when personal stories establish a process of identification with family histories. In this panorama, based on a review of the literature on succession processes in the field of family businesses and on organizational identity, some reflections are constructed that can generate future research. This is a study that does not fit into the line of functionalist studies, because it raises reflections from the intersubjective dynamics.
\end{abstract}

Keywords: Organizational Identity. Family business. Succession Processes

\section{RESUMO}

Este estudo sustenta que a sucessão em uma empresa familiar também é uma questão de identidade organizacional. Nosso argumento baseia-se na ideia de que a sucessão nos negócios da família é possível quando há um forte processo de identificação com a empresa, que não está necessariamente vinculado unilateralmente à história da empresa familiar e aos laços familiares diretos, mas pode existir quando histórias pessoais estabelecem um processo de identificação com histórias familiares. Nesse panorama, baseado em uma revisão da literatura sobre processos de sucessão no campo das empresas familiares e sobre identidade organizacional, são construídas algumas reflexões que podem gerar pesquisas futuras. Trata-se de um estudo que não se enquadra na linha dos estudos funcionalistas, pois suscita reflexões a partir da dinâmica intersubjetiva.

Palavras-chave: Identidade Organizacional. Empresas Familiares. Processos de Sucessão

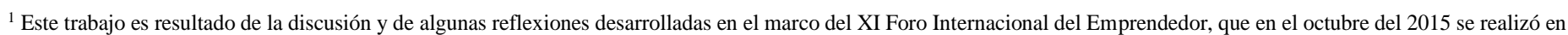
Manizales, Colombia, y enfocaba el tema de la Empresa Familiar, Emprendimiento y PyME y actualizado con revisión bibliográfica sobre el tema.

${ }^{2}$ Mariana Lima Bandeira. Doctora en administración de la Fundación Getulio Vargas, profesora titular de la Universidad Andina Simón Bolívar, profesora invitada de la Universidad Estatal de Milagro. mariana.lima@uasb.edu.ec.

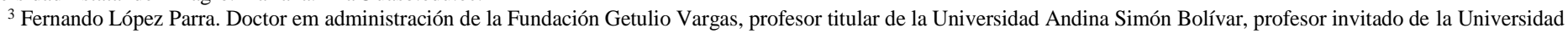
Federal Fluminense, Rector del Instituto de Altos Estudios Nacionales. fernando.lopez@uasb.edu.ec.

${ }^{4}$ Marcelo Reinosos Navarro. Maestro en Gerencia de proyectos por la EPN y BID, profesor de posgrado en la UASB y en el Instituto de Altos Estudios Nacionales. marcelo.reinoso@iaen.erdu.ec.
} 


\section{Introducción}

La empresa familiar es una modalidad de emprendimiento muy encontrada en todo el mundo y, por su rol en la economía y en la regulación de la dinámica socio-institucional, ha venido adquiriendo importancia también en los estudios organizacionales (Teixeira 2011). Pese que a una primera vista los campos de emprendimiento y de negocios familiares podrían haberse desarrollado de forma independiente, los estudios sobre esta articulación han crecido y podrían representar al menos un 5\% de la producción académica actual (López-Fernández, Serrano-Bedia \& Pérez-Pérez, 2015).

Estudios bibliométricos comprueban el creciente interés en estudiar este campo (Paiva, Oliveira y Melo, 2008; Martins et al, 2012; Alberto, Famá \& Junqueira, 2013; Vicente et al, 2015; Mayer \& Werlang, 2016). Adicionalmente, también los levantamientos realizados por observatorios y organizaciones sin fines lucrativos - o similares - también refuerzan la importancia de los negocios familiares, en todo el mundo: PWC, KPMG, European Family Businesses, Family Firm Institute, Instituto de Empresa Familiar (España), Asociación Murciana de la Empresa Familiar (España), Instituto de Desenvolvimento da Empresa Familiar (Brasil), Instituto Ecuatoriano de Gobernanza Corporativa (Ecuador), entre otros.

La gobernanza de una empresa familiar muchas veces se ve afectada por problemas de orden subjetivo, a tal punto que su propia sustentabilidad podría estar en riesgo. Los problemas se tornan visibles en momentos de crisis, algunos de ellos cuando se plantea la sucesión. Esta realidad no es nueva, Donneley $(1964,1967)$ ya la reconocía como uno de los "peligros" de la falta de claridad entre lo familiar y lo empresarial de una empresa familiar. Estudios actuales todavía muestran que este no es un problema que ha tenido una solución legítima, pues las propuestas siguen en construcción, desde distintos abordajes (Rodrigues, 1991; Rossato Neto \& Cavedon, 2004; Tillmann \& Grzybovski, 2005; Eccel, Cavedon \& Craide, 2007; Gómez-Betancourt, Betancourt-Ramírez \& Zapata -Cuervo, 2012; Madison et al., 2016).

Los modelos de planificación para la sucesión generalmente valoran elementos de gobernanza muy concretos, que podrían ser medibles, y entre los más frecuentes están la transmisión del patrimonio, del poder decisorio y del conocimiento, la definición del rol de los propietarios y de los gestores de la empresa, la flexibilización de la estructura de gobierno para descentralizar decisiones, equilibrar intereses de cada actor y promover una transición mínimamente traumática para la sucesión.

Pese los distintos abordajes y numerosas propuestas, los números en Ecuador muestran que estos esfuerzos no son totalmente efectivos. De acuerdo al Instituto Ecuatoriano de Gobernanza Corporativa, 67\% de las empresas familiares son de primera generación, el 23\% de segunda generación, el $7 \%$ de tercera generación y apenas el 3\% cuarta generación o más (Villagómez, 2015).

En este artículo, se propone que la sucesión en una empresa familiar ha olvidado de una dimensión simbólica muy importante: el establecimiento de un proceso de identificación con la filosofía de la empresa. Es decir, la sucesión es, también, una cuestión de identidad organizacional.

Nuestro argumento se construye a partir de la idea de que la sucesión en la empresa familiar es posible cuando hay un fuerte proceso de identificación con la empresa. Este proceso de identificación no necesariamente está vinculado unilateralmente a la historia de la empresa familiar y a los lazos familiares directos, puede existir cuando las historias personales establecen un proceso de identificación con las historias de la familia. En este panorama, la complejidad se agudiza si consideramos la convivencia intergeneracional y como los distintos valores de cada generación puede influir en las generaciones de la empresa familiar.

De esta manera, se pretende justificar este argumento a partir de una revisión de la literatura sobre la dinámica de identificación entre los miembros de la empresa familiar, considerando ser una perspectiva compleja y articulada a elementos antropológicos, psicosociales, económicos y también de la historia familiar particular de cada empresa.

La pregunta que dirigió esta investigación puede ser presentada de la siguiente 
manera: ¿Cómo se configura la identidad organizacional en una empresa familiar en el contexto de la sucesión para la sustentabilidad del negocio?

Se trata de una revisión bibliográfica, documental y uso de datos secundarios, a partir de los cuales se construyen algunas reflexiones que podrían generar futuras investigaciones. Es un estudio que no se inscribe en la línea de estudios funcionalistas, toda vez que no busca traer una receta, más bien invita a una reflexión desde dinámicas intersubjetivas.

A partir de lo expuesto, este artículo se organiza en cuatro sesiones. La primera y la segunda tratan de explorar los conceptos y las dinámicas de la empresa familiar y la problemática para construir los procesos sucesorios. En seguida, se destina parte del corpus para traer la complejidad de la construcción de una identidad organizacional. La cuarta y última sesión explora esta construcción en el ámbito de las empresas familiares, buscando una deconstrucción de las dinámicas de los procesos sucesorios a partir de elementos exclusivamente instrumentales y tejiendo algunas reflexiones sobre ello.

\section{Empresas familiares: conceptos ampliados}

Robert Donnelley, uno de los estudiosos precursores en esta temática, considera una empresa como familiar cuando ha estado ligada a una familia por al menos "durante dos generaciones y con ligaciones familiares que ejerzan influencia sobre las directrices empresariales, los intereses y objetivos de la familia" (Donnelley, 1967, p. 161). En este sentido, la naturaleza de una empresa familiar se caracteriza por considerar el factor hereditario para la sucesión del directorio (Donneley, 1967).

La delimitación de un concepto claro de la empresa familiar es una tarea compleja y, conforme algunos autores afirman, se debe principalmente al hecho de que son realidades multidimensionales por naturaleza (Arenas-Cardona y Balvín, 2014). Considerando sus ambigüedades, hay alguna divergencia en la literatura sobre cuáles se pueden considerar una empresa familiar. Incluso hay la perspectiva de que una organización puede ser adjetivada como familiar cuando la relación entre los miembros está pautada por "valores de familia": dedicación, afectividad, respeto, entre otros (Bernhoeft, 1989; Paiva, Oliveira y Melo, 2008). Otros proponen la separación de propiedad y gestión - la familia es propietaria pero no administran la empresa directamente (Lethbridge, 1997).

Ampliando el concepto de empresa familiar, podemos entonces sintetizar que es un emprendimiento compuesto de múltiples miembros de la misma familia, como propietarios o gerentes, de una o más generaciones o que, a lo mejor, es un conjunto de personas que se sienten de la familia y comparten los valores familiares; la familia - con sus miembros legítimos o no - controla el negocio a través de posiciones de propietarios y gestores, con distintos porcentajes de participación y tienen interés en dar continuidad al negocio, estableciendo un compromiso y un vínculo emocional; generalmente las decisiones son centralizadas en la persona que estableció la empresa o sus familiares directos y al menos un representante familiar está al frente de decisiones de gobernanza.

Esta modalidad de emprendimiento siempre ha tenido su importancia en la configuración de la economía mundial y no se trata apenas de pequeños y medianos emprendedores. Sus definiciones y delimitaciones conceptuales conllevan a afirmar que no existe un consenso en la literatura (Tillmann \& Grzybovski, 2005), y se puede encontrar más de 34 conceptos distintos (Davel \& Colbari, 2000).

Algunos investigadores identifican tres dimensiones que definen la particularidad de un negocio familiar, que son la familiar, la gerencial y la propiedad (Astrachan, Klein \& Smyrnios, 2002; Schmidts \& Shepherd, 2013; Arenas y Balvín, 2014). No es difícil entender que estas tres dimensiones muchas veces se confunden en la gestión del negocio, generando resultados para la sustentabilidad ora positivos y ora negativos (Poza, 2007, citado por Arenas y Balvín, 2014).

Una combinación armónica entre estos tres pilares podría canalizar a una reducción de los conflictos internos $\mathrm{y}$, por ende, de los problemas que se originan en estos conflictos (Schmidts \& Shepherd, 2013). Algunos debates recientes ilustran que es posible una combinación entre los sistemas familiares y de la empresa, en la cual existen convergencias entre los objetivos de ambas las partes. De acuerdo con Schmidts \& Shepherd (2013), los 
resultados de la investigación de Randel Carlock y John Ward, publicados en el 2001, sostienen el argumento de que debe existir un equilibrio entre ambos sistemas. En el caso de haber una priorización de la dimensión de negocios, podría haber como resultado un deterioro de las relaciones familiares y el contrario también podría darse: caso se sobrevalore las relaciones familiares, el desempeño, la toma de decisiones, las opciones estratégicas y los resultados organizacionales.

Las contradicciones también se pueden observar en la literatura. Un estudio realizado por Santiago Dodero, entre el 2002-2005, a 661 miembros de empresas familiares latinoamericanas muestra estas ambigüedades. Por un lado, los valores, la dedicación y la unidad son considerados factores de éxito; por otro lado, la falta de compromiso, los conflictos por fallas de comunicación y la superposición de roles son errores que aparecieron en la investigación (Dodero, 2005).

Contrariamente al imaginario popular de la existencia de grandes corporaciones, la mayoría de empresas en el mundo están controladas por familias o por el Estado (Paiva, Oliveira y Melo, 2008). Se evidencian con datos estadísticos que grandes empresas son caracterizadas por ser familiares y representan una participación importante en el dinamismo de la economía. Un levantamiento hecho en Brasil, al final de los años 90, indica que en este entonces "casi 90\% de los 300 mayores grupos nacionales privados son administrados por miembros de la familia controladora, generando un total de 1,6 millones de empleos directos" (Souza-Silva, Fischer y Davel, 1999, p.2). Por otro lado, las pequeñas y medianas empresas también se caracterizan por ser empresas de familia o familiares (Mayer \& Werlang, 2016).

Los problemas más comunes en las empresas familiares y que afectan la sustentabilidad del negocio son los siguientes:

Crecimiento e Internacionalización - Actualmente, el 25\% de los ingresos de las empresas familiares de todo el mundo viene de las exportaciones. Un peso que esperan aumentar hasta el 32\% en los próximos cinco años.

\section{- Profesionalización y Desarrollo del Talento Humano}

- Solución de conflictos internos - en relación a visiones, opiniones, decisiones, potenciados por distintas generaciones.

- Preparar un Plan de Sucesión que pueda ser aplicado por y entre las distintas generaciones de familia y de la empresa familiar.

En este capítulo, nos interesa especialmente los tres últimos elementos que, en principio, se podrían convertirse en un solo: la cuestión de la gobernanza. Daremos especial énfasis a la sucesión familiar en el ámbito de la gobernanza, en el siguiente acápite.

\section{La sucesión en la empresa familiar}

El proceso sucesorio es de mucha relevancia en cualquier tipo de empresa, sea familiar o no, pero es necesario destacar que, en el caso de las empresas familiares, a las complejidades propias de cualquier proceso sucesorio, se añaden las tensiones emocionales particulares que se generan en el seno familiar (Flores Jr \& Grisci, 2012) y que se proyectan en una mayor o menor identificación de las personas con el negocio (Gómez-Betancourt, Betancourt-Ramírez \& López-Vergara, 2013).

La sucesión es un elemento central para el mismo concepto de empresa familiar, ya que apenas después de la segunda generación asumir el comando es que se puede considerar una empresa como familiar (Donneley, 1967). Hasta mientras, es una empresa de la familia ((Tillmann \& Grzybovski, 2005). La sucesión, en el contexto de una empresa, es planificada a partir de documentos y directrices que organizan la transferencia de la dirección de una empresa. En el caso de una empresa familiar, también dice respeto a la transferencia de la propiedad de la firma (Arenas-Cardona y Balvín, 2014).

Este proceso implica que se usen varias herramientas como protocolos, técnicas de gobiernos corporativos / gobiernos familiares, planes de sucesión (Arenas-Cardona y Balvín, 2014), los cuales se deben diseñar en función de las características particulares de cada organización, así como de la configuración del torno en el que desarrollan sus actividades. Es 
esperado que el protocolo pueda mantener "la cohesión y la armonía familiar" (p. 254) siempre cuando sea elaborado en momentos de unidad familiar y empresarial -, regular el patrimonio y permitir una transición mínimamente equilibrada en términos de valores, significados y estrategias, por lo que "la sucesión no puede ser vista como 'una fase', pero como un conjunto de estrategias deliberads en el seno familiar y, en momento oportuno, presentadas a la empresa" (Tillmann \& Grzybovski, 2005, p. 48).

Sin embargo, por diversos motivos, la acción de sucesión no se la ejecuta o no se lo hace de acuerdo al planificado. Los planes de sucesión implican una planificación ordenada en términos de documentos y propiedad, pero también incluye la transición del liderazgo, siendo considerado un proceso que debe ser llevado durante cierto tiempo (Arenas-Cardona y Balvín, 2014). Además, se evidencia que cada cambio sucesorio incrementa el número de propietarios (Betancourt-Ramírez, Gómez-Betancourt \& López-Vergara, 2011).

La sucesión en una empresa familiar viene siendo investigada considerando su proceso, su gobernanza y modelo de gestión y temas de profesionalización. Otra situación poco tratada en los estudios es el problema de la falta de sucesor (Mayer \& Werlang, 2016). Algunas veces, en pequeños o grandes emprendimientos, los posibles sucesores eligen otras trayectorias profesionales muy distintas del camino recogido por sus padres $y$, consecuentemente, se distancian del negocio familiar.

Como es de esperar, la sucesión en una empresa familiar es un tema complejo y que no implica apenas un cambio en la dirección de la empresa: se produce cambios en la propiedad, en la estrategia del negocio, en la estructura y dinámica familiar y, también, de la empresa, impactando en el comportamiento del consumidor/cliente y también en los funcionarios y en su gobernanza interna (Santos et al., 2016).

Un levantamiento realizado por Ernest \& Young ${ }^{5}$, de 2015, se deduce que la sucesión sigue siendo el mayor problema de las empresas familiares:

La mayoría de ellas siguen en manos de la primera generación, aunque hay casos excepcionales. El 53\% de los líderes que participaron en la encuesta pertenecen a la segunda generación o, incluso, a alguna superior. El caso más llamativo lo protagoniza Alemania.

Las empresas familiares alemanas están dirigidas mayoritariamente por la tercera y la quinta generación (así lo reconocieron un 25\% de los encuestados en cada caso), pero tienen también empresas lideradas por familias que pertenecen ya a la sexta generación (6\%), séptima (13\%) e incluso a la novena (6\%). Algo insólito en el resto de los países que se analizan en el estudio.

En esta misma línea de argumentación, se identifica que la falta de preparación previa a la sucesión genera varios problemas de distintas índoles y naturaleza (Santos et. al, 2016). También se evidencia que, cuando cambia el líder, hay una mudanza cultural, lo que muchas veces dificulta el relacionamiento con sus demás actores (clientes internos, externos, stakeholders, etc.), y no apenas del negocio en sí mismo.

Además de la falta de preparación para la sucesión, también se encontró que el sucesor no siguió los pasos de su antecesor y un tema que también se mostró tanto en el estudio de Santos et al (2016) cuanto en el estudio de EY (2015) fue el tema de género. Si bien el estudio en Brasil indica que las mujeres no tienen las mismas oportunidades para participar en el proceso decisorio, el estudio de EY (2015, p. 4), señala que: "el 70\% de los grandes negocios familiares están considerando nombrar a una mujer como próximo CEO. España y Alemania son los que están más conformes con esta idea”.

Paralelamente, en los últimos años, de acuerdo a datos levantados por Villagómez (2015), al menos medio millón de empresas desaparecieron en Sudamérica por falta de preparación de la sucesión y el 95\% de las empresas tienen reglas informales o no tienen reglas en el proceso de sucesión. Esta realidad también se reproduce en Ecuador y, conocida estas características, es posible entender por qué, en el país, apenas el 33\% de las empresas familiares llegan a la $2^{\mathrm{a}}$ generación, menos del $15 \%$ llegan a la $3^{\mathrm{a}}$ generación y apenas el $4 \%$

\footnotetext{
${ }^{5}$ Encuesta realizada en 2014 a las 2.400 mayores empresas familiares del mundo procedentes de los siguientes países: Australia, Bélgica, Brasil, Canadá, China, Francia,

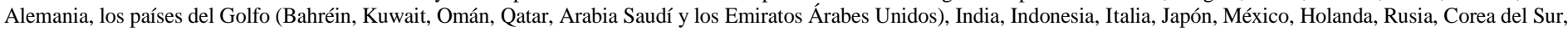
España, Suiza, Turquía, Reino Unido y EEUU.
} 
llega a la cuarta generación (Instituto Ecuatoriano de Gobernanza Corporativa, 2015).

Desde un enfoque teórico-empírico, se puede decir que la sucesión consiste en un proceso dinámico mediante el cual los roles del predecesor y sucesor evolucionan independientemente $y$ en paralelo hasta que finalmente se traslapan y confunden provocando un conjunto de situaciones complejas. La próxima sección del capítulo trata de abordar esta complejidad desde la perspectiva de la identidad organizacional, buscando establecer conexiones entre las fronteras entre los distintos dominios: de familia y de empresa, que deben equilibrar sus actores - miembros o no de la familia.

\section{La identidad organizacional}

La identidad organizacional no es un conjunto de identidades individuales dentro o fuera de una organización (Kreiner, 2011); tampoco se puede traducirla como identidad corporativa, un concepto que viene del marketing y que está enfocado a la imagen de la organización que es comunicada a sus distintas audiencias.

Desde las corrientes tradicionales, se podría afirmar que la perspectiva del construccionismo social y del actor social son dos ejes importantes que sostienen las ideas desarrolladas en torno a la identidad organizacional. Kreiner (2011), analizando estos aportes, busca identificar las relaciones entre los conceptos de identidad y cultura organizacional, estableciendo una estrecha relación entre ambos. Para la autora, "ambos se (rely) en valores y supuestos, y aunque talvez de difícil acceso, ambos son herramientas poderosas para la comunicación y acción” (Kreiner 2011, p. 463-464).

Kreiner (2011) evidencia que el interés en entender el tema se ha acentuado a partir de los años 2000, pese a que Stuart Albert y David Whetten, en 1985, introdujo el concepto de identidad organizacional. Actualmente, se observan algunos elementos interesantes de reflexión y avances teóricos, pero todavía falta investigación empírica que legitime lo suficientemente bien las proposiciones.

Por otro lado, Thomas (2011) está preocupado en analizar los procesos intersubjetivos y las interrelaciones entre poder y subjetividad en la dinámica de formación de la identidad organizacional. Este enfoque podría entregar a nuestra investigación una riqueza en la discusión de la convivencia intergeneracional y como estos procesos pueden fortalecer ciertos valores y, a la vez, transformar otros, en la construcción de la identidad organizacional de una empresa familiar.

En su problematización, el autor sugiere que el sistema capitalista podría ser responsable por un incremento en la inseguridad del empleo, fomentado la movilidad y corrompido muchos de las muchas anclas tradicionales de la identidad, como la familia, trabajo, vecindad, y a la final, creado una crisis de identidad. Es importante hacer hincapié en como la familia, en sociedades más tradicionales como la ecuatoriana, puede ejercer fuerte influencia en la construcción de identidad.

Asimismo, en esta línea de argumentación, los miembros de una empresa familiar ejercen su posición de sujeto, construyendo y reconstruyendo su subjetividad. La identificación y la identidad, en el contexto social familiar, se mezclan como procesos similares. Sin embargo, sus particularidades se evidencian, principalmente cuando se abordan los conflictos y las divergencias en la dinámica sucesoria.

El vínculo que se presenta entre los miembros de la empresa familiar está fundamentado también por el conjunto de valores y principios construidos en el seno de la familia y reproducidos en la dinámica organizacional (Tillmann \& Grzybovski, 2005). Sin embargo, estos vínculos se mezclan con la dinámica del mercado y algunas veces las distintas generaciones que interactúan en una empresa familiar pueden divergir en estos aspectos. Estas rupturas, propias de las construcciones intersubjetivas, podrían provocar cambios en la identidad organizacional.

Esto es lo que plantean Sundaramurthy \& Kreiner (2008), al usar la "teoría de fronteras" para intentar explicar el grado de permeabilidad y de flexibilidad entre los roles que desempeñan los miembros de una empresa familiar, en los distintos ámbitos. Esto implica entender cuando ser flexible (adaptarse, abriendo mano de un rol, por ejemplo), cuando permanecer inquebrantable y cuando asumir la posición hibrida en relación a los 
roles (integrarlos).

Una forma de construcción temprana de los vínculos de los sucesores con el negocio familiar es insertarlos precozmente - ya desde la temprana edad - en la dinámica organizacional, estableciendo procesos simbólicos de identificación, "haciéndolos aprender a amar a la empresa" (Tillmann \& Grzybovski, 2005, p. 52).

La identidad también se expresa en el espacio de trabajo, constituyéndose en la dimensión territorial y espacial del individuo en su comportamiento en las organizaciones (Fischer, 1994). Esta expresión se manifiesta de acuerdo a la naturaleza del trabajo, al sentimiento de estabilidad del y en el grupo y la personalidad del individuo (Sundstrom \& Sundstrom, 1986). La personalización de este espacio intuye una autonomía y una seguridad em el rol desempeñado em el grupo y de su grado de pertenencia. Esto es lo que también asume Jean François Chanlat, al defender la idea de que "el ser humano se enraíza en el tiempo y en el espacio" (Chanlat, 1996, p. 108). El mencionado autor aclara que estas dimensiones están siendo rescatadas en el marco del comportamiento humano y no desde una visión funcionalista. De esta manera, el individuo busca se apropiar de los símbolos que lo identifican y este espacio se organiza en un "campo que estructura las interacciones" (Chanlat, 1996, p.108), siendo parte también de su subjetividad.

Borzeix \& Linhart (1996) analizan un caso interesante en Francia donde, impulsadas por una ley, las empresas instituyeron las llamadas "reuniones de expresión" por medio de "interlocutores colectivos", que serían supuestamente representativos de grupos de individuos en las organizaciones. De este análisis, lo que es interesante hacer hincapié en el marco de nuestro argumento es que la familia, como una identidad colectiva, puede presentar las mismas dificultades en el proceso de representación de valores, ideas e ideales grupales.

Uno de los hallazgos de esta experiencia que pueden servirnos como punto de reflexión es que la representación por medio del interlocutor colectivo no avanzó para la construcción de una consciencia propia y autónoma del grupo, no produjo otros actores y tampoco ayudó a reducir la tensión entre lo individual y lo colectivo. Transfiriendo esta perspectiva al argumento que queremos defender en este capítulo, podemos abstraer que los interlocutores principales de la familia no tienen la plena capacidad de responder por todos los demás. Su acción es limitada y, en este caso, los demás miembros de otras generaciones podrían no tener la misma visión, los mismos valores y el mismo compromiso con la sustentabilidad de la empresa.

Al final, también se plantea que la familia (como ente colectivo), en sí mis ma, no tiene la capacidad de representar los intereses, las demandas y los ideales de sus miembros en la dimensión individual. Al mismo tiempo, el interlocutor individual tampoco podría equilibrar las distintas demandas del grupo.

Esta plasticidad de identidades colectivas (Borzeix \& Linhart, 1996) se observa en el concepto tradicional de familia y, por ende, se transfiere a la empresa familiar con bastante fuerza, ya que la construcción de identidades transita entre conflictos, ausencias, fragmentaciones, temporalidades y diversidades de expresión de la identidad.

A esta altura, se podría recuperar la pregunta que Martin Parker hace en el inicio de su libro, en el que cuestiona si las organizaciones pueden moldear la identidad de sus miembros (Parker, 2000). Es oportuno también ampliar la reflexión, planteando si no son los mismos individuos que construyen la identidad de la organización a que pertenecen, en mayor o menor grado.

Parker (2000) es crítico con la literatura sobre cultura organizacional desarrollada hasta entonces, y le califica como poco reflexiva, toda vez que se olvida de sus premisas centrales. El autor propone que la cultura organizacional debe ser vista como unidades fragmentadas y con las cuales sus miembros, ora se identifican como entes colectivos, ora lo hacen como seres individuales.

Uno de los ejemplos que da fuerza a su propuesta es la descripción de un caso de las Industrias Vulcan, en que Parker (2000) empieza con la presentación del discurso "oficial" por parte de los gerentes, para quienes la empresa es una familia: "con un lenguaje común, tradiciones y una identidad compartida" (Parker, 2000, p. 127). Para dar sustento a su argumento, Parker (2000) presenta las diferencias y fragmentaciones de la cultura de la 
empresa, a partir de categorías binarias a las que considera inseparables: los de arriba vs los de abajo, dos tecnologías, los nuevos y los viejos ingenieros, mi departamento vs su departamento, juntos y separados, entre otras. Estas categorías visibilizan las disputas, los conflictos y la dinámica social de establecimiento de las identidades sociales.

El abordaje de Thomas (2011) está enfocado en señalar los avances del tema en el marco de los estudios críticos de la gestión y excluye de su propuesta las perspectivas de la teoría de la identidad social de Tajfel and Turner (1979). Para el autor, esta visión apenas profundiza el enfoque funcionalista de la gestión, toda vez que pone la organización como referencia central en la definición de identidades de los individuos. A partir de este supuesto, sugieren los autores que se podría alcanzar una congruencia importante entre los objetivos organizacionales e individuales, incrementando los compromisos, la lealtad y la motivación.

\section{Algunas reflexiones finales}

Esta sección está dedicada a tejer algunas articulaciones entre el proceso sucesorio de empresas familiares y la construcción de identidad (es), a partir de las temáticas teóricas que fueron desarrolladas previamente.

Generalmente, en los planes de sucesión, cuando los hay, se establecen objetivos, estrategias, procesos y metas de forma muy directa. Se supone que los protocolos de sucesión dan pautas seguras para la gobernanza de una empresa familiar y su sustentabilidad. Asimismo, se asume que serán aplicados fielmente y darán los resultados esperados. Sin embargo, no consideran la dinámica de la familia misma, sus elementos subjetivos y procesos de identificación que, muchas veces, apelan para una dimensión emocional y simbólica. En este sentido, las contradicciones entre el plan y la vida organizacional se refuerzan a lo largo del tiempo.

Es conocido que los planes de sucesión o protocolos logran resultados siempre cuando los miembros de las distintas generaciones se apropian - incluso emocionalmente - de la identidad organizacional. Algunas aproximaciones empíricas realizadas para la confección de este capítulo nos muestran que es posible encontrar la necesidad de perpetuar el negocio familiar sin, con todo, que la misma familia esté involucrada. Existen cambios en el proceso de identificación de las distintas generaciones y que indubitablemente esta dinámica impacta en la sucesión de la empresa familiar. Distintas generaciones, creadas en diferentes contextos, poseen sueños, proyectan imaginarios y tienen aspiraciones que pueden distanciarse de los planes de permanencia de las empresas de sus familias. Las trayectorias profesionales no se definen a partir de la familia, más bien pueden tener otros focos de interés.

La sucesión de una empresa familiar demanda un alineamiento o una articulación con el sueño inicial de su fundador, con los principios adoptados, los valores y símbolos construidos en la familia y que se consolidan en la empresa. Incluso las prácticas de gestión están impregnadas con el estilo de la familia. Por lo tanto, se torna un gran desafío la construcción de una identidad familiar-organizacional en el ámbito de distintas generaciones.

Las nuevas generaciones tienen influencia del entorno y, supuestamente, para dar continuidad al negocio familiar, deberían seguir las tendencias de formación más modernas para dar soporte y actualizar la empresa familiar. Sin embargo, hay otras perspectivas de análisis. Podemos encontrar que más importante es el componente colectivo que el individual, cuando se decide por mantener y dar continuidad al negocio familiar.

\section{Referencias bibliográficas}

Alberto, G. S. Famá, R. \& Junqueira, L. A..P. (2013). Governança corporativa em Empresas Familiares: um estudo bibliométrico e análise de redes sociais. Revista Eletrônica de Administração, v. 12, n.2, ed. 23, jul-dez 2013. ISSN: 1679-9127

Arenas-Cardona, H. A. Balvín, D. R. (2014) La empresa familiar, el protocolo y la sucesión familiar. Estudios Gerenciales, Vol. 30, p. 252-258. 
Astrachan, J. H., Klein, S. B., Smyrnios, K. X. (2002) The F-PEC Scale of family influence: A proposal for solving the family business definition problem. Family Business Review, Vol. 15, No. 1, pp. 45-58.

Bernhoeft, R. (1989) Empresa familiar: sucessão profissionalizada ou sobrevivência comprometida. São Paulo: Nobel.

Betancourt-Ramírez, J. B., Gómez-Betancourt, G. López-Vergara, M. P. (2011) Aproximación a los factores que influyen en la visión del patrimonio en las empresas familiares colombianas. Estudios Gerenciales, Vol. 120, No. 27, p. 165-184.

Borzeix, A. Linhart, D. (1996). Identidades e práticas linguísticas na empresa. En: Chanlat JeanFrançois. O indivíduo na organização: dimensões esquecidas. Vol. III. São Paulo: Atlas. (p.81-106).

Chanlat, J. F. (1996). O ser humano, um ser espaço-tempo. En: Chanlat Jean-François. O indivíduo na organização: dimensões esquecidas. Vol. III. São Paulo: Atlas. (p.107-110).

Dodero, S. (2005) Investigación sobre la empresa familiar en Latinoamérica. ADEN-Instituto de la Empresa Familiar. Disponible en: http://biblioferrersalat.com/media/documentos/Investigacion\%20sobre\%20la\%20Empresa\%20Familia r\%20en\%20Latinoamerica\%20(2).pdf Visitado en 08 de junio del 2018.

Donneley, R. G. (1967) A empresa familiar. RAE-Revista de Administração de Empresas, vol. 7, n. 23, abr-jun 1967, pp. 161-198. Traduzido do inglês por Carlos Osmar Bertero e publicado mediante autorização da Harvard Business Review, Vol. 42, n.? 4, julho-agosto de 1964, onde foi originalmente publicado.

Eccel, C. S. Cavedon, N. R. Craide, A. (2007). A empresa familiar Cachorro Quente do Rosário: entre a antropología e a administracao. Organizações e Sociedade - O\&S - v.14 - n.42 - Julho/Setembro, p. 49-66.

EL COMERCIO. Redacción Negocios: "9 de cada 10 empresas en el Ecuador son familiares". Publicado el 21 de noviembre del 2017. Disponible en: http://www.elcomercio.com/actualidad/empresas-ecuadorfamilia-manufactura-agricultura.html

EL TELÉGRAFO. Redacción Economia: "En el país, el 90,5\% de empresas son familiares. 28 de noviembre del 2017. Disponible en: https://www.eltelegrafo.com.ec/noticias/economia/1/en-el-pais-el90-5-de-empresas-son-familiares Visitado en abril del 2018.

EUROPEAN FAMILY BUSINESSES - 2012. Datos disponibles em: http://www.europeanfamilybusinesses.eu/family-businesses/facts-figures

EY - Ernest Young. Centro de Estudios EY. Informe global sobre las mayores Empresas Familiares. Resumen Ejecutivo. 2015. Disponible em: http://www.ey.com/Publication/vwLUAssets/EY-informeglobal-sobre-las-mayores-empresas-familiares-resumen-ejecutivo/\$FILE/ey-informe-global-sobre-lasmayores-empresas-familiares-resumen-ejecutivo.pdf

Fischer, G. N. (1994) Espaço, identidade e organização. En: Chanlat, Jean-François. O indivíduo na organização: dimensões esquecidas. Vol. II. São Paulo: Atlas. (p.81-102).

Flores Jr., J. E. Grisci, C. L. I. (2012). Dilemas de pais e filhos no processo sucessório de empresas familiares. Revista de Administração, São Paulo, Vol. 47, No. 2, p. 325-337, abr./maio/jun.

Gómez-Betancourt, G. Betancourt-Ramírez, J. B. López-Vergara, M. P. (2013) Factores que influyen em la inteligência emocional de los membros de uma empresa familiar. Entramado, Vol. 9, No. 1 (Enero-Junio), p. 17-25.

Gómez-Betancourt, G. Betancourt-Ramírez, J. B. Zapata-Cuervo, N. (2012). Empresas familiares multigeneracionales. Entramado Vol. 8 No. 2 (Julio - Diciembre), p. 38-49.

Hernández-Linares, R. Soumodip, S. López-Fernández, M. C. (2017) Concepción. How has the family firm literature addressed its heterogeneity through classification systems? An integrated analysis; Cómo ha abordado la literatura de la empresa familiar su heterogeneidad a través de sistemas de clasificación? Un análisis integrado. European Journal of Family Business, Vol. 7, No. 1-2, JanuaryDecember, p.1-13. Research Paper. DOI: https://doi.org/10.1016/j.ejfb.2017.06.003

Kreiner, G. E. (2011) Organizational Identity: culture's conceptual cousin. In: Ashkanasy, Neal M., Wilderom, Celeste P. M., Peterson, Mark F (Eds.). The Handbook of Organizational Culture and Climate. 2nd Edition. Thousand Oaks, CA, USA: Sage. Chapter 26. 463-480.

Lethbridge, E. (1997) Tendências da empresa familiar no mundo. Revista do BNDES, Rio de Janeiro, v. 4, n. 7, p. 185-200, jun.

López-Fernández, M. C. Serrano-Bedia, A. M. Pérez, M. (2016) Entrepreneurship and Family Firm 
Research: A Bibliometric Analysis of An Emerging Field. Journal of Small Business Management, Vol. 54, No. 2, April, p. 622-639. (First published: 18 March 2015). DOI: https://doi.org/10.1111/jsbm.12161

Madison, K. Holt, D. T., Kellermanns, F. W. \& Ranft, A. (2016) Viewing family firm behavior and governance through the lens of Agency and Stewardship Theories. Family Business Review, Vol. 29, No. 1, p. 65-93. DOI: DOI: 10.1177/0894486515594292

Martins, R. A. Serra, F. A. R. Ferreira, M. P. Reis, N. R. (2012) Análise da produção científica sobre empresas familiares: um estudo bibliométrico. XXXVI Encontro da ANPAD, Anais... Rio de Janeiro, 22 a 26 de setembro de 2012 .

Mayer, C. E. Werlang, N. B. (2016) Mapeamento bibliométrico a partir do contruto de sucessão. I Workshop de Práticas Tecnológicas no Agronegócio. Memórias, 2016. Disponible en: https://eventos.uceff.edu.br/eventosfai_dados/artigos/inovaagro2016/581.pdf

Nandi, R. (2017) Learning to share family business identity with non-family members. AI Practitioner, Vol. 19, No. 4, november. ISBN 978-1-907549-33-5. DOI: dx.doi.org/10.12781/978-1-907549-33-5-20

Paiva, K. C. M. OLIVEIRA, M. Melo, M. C. (2008) Produção científica brasileira sobre empresa familiar - um metaestudo de artigos publicados em Anais de eventos da ANPAD no período de 1997 -2007. RAM - REVISTA DE ADMINISTRAÇÃO MACKENZIE, V. 9, N. 6 • P. 148-173 • SET./OUT. • ISSN 1678-6971

Parker, M. (2000). Organizational culture and identity: unity and division at work. London: SAGE, 2000.

Rodrigues, A. M. (1991) Padrões afetivos na família e empresa familiar. Revista de Administração de Empresas - RAE, São Paulo, Vol 31, No. 4, Out. Dez. p. 35-48.

Rossato Neto, F. J. CAVEDON, N. R. (2004) Empresas familiares: desfilando seus processos sucessórios. Cadernos EBAPE.BR, Vol. II, No. 3 - Dezembro, p. 1-16

Santos, A. A. P. Sehnen, S. Casagrande, J. L. Schutz, E. (2016) Sucessão familiar: uma análise dos escritos brasileiros do período de 2004 a 2015. XIX SEMEAD Seminários em Administração, São Paulo, novembro. ISSN 2177-3866

Schmidts, T. Shepherd, D. (2013) Social identity theory and the family business: a contribution to understanding family business dynamics. Small Enterprise Research, Vol. 20, No. 2, p. 76-87.

Souza-Silva, J. C.; Fischer, T., Davel, E. (1999) Organizações familiares e tipologias de análise: o caso da Organização Odebrecht. In: ENANPAD, 23., Foz do Iguaçu. Anais... Foz do Iguaçu: Anpad, 1999.

Sundaramurthy, C. Kreiner, G. E. (2008) Governing by managing identity boundaries: the case of Family businesses. Entrepreneurship Theory \& Practice, May, p.415-436.

Sundstrom, E. Sundstrom, M. G. (1986) Work places: the psychology of the physical environment in offices and factories. New York: Cambridge University Press.

Tajfel, H. Turner, J. (1979) An integrative theory of intergroup conflict. In W. G. Austin, \& S. Worchel (Eds.), The social psychology of intergroup relations (p. 33-37). Monterey, CA: Brooks/Cole

Teixeira, A. (2011). Mapping the (In)visible Collage(s) in the Field of Entrepreneurship. Scientometrics 89, p. 1-36.

Thomas, R. (2011) Critical management studies on identity: mapping the terrain. In: Alvesson, Mats. Bridgman, Todd. Willmott, Hugh (Eds.). The Oxford handbook of Critical Management Studies. Oxford University Press. Chapter 8, p. 166-185.

Tillmann, C. Grzybovski, D. (2005) Sucessão de dirigentes na empresa familiar: estratégias observadas na família empresaria. Organizações \& Sociedade - O\&S, Vol. 12, no. 32, Janeiro/Marco, p. 45-61.

Tsai, F. S; Lin, C.H., Lin, J.L. Lu, I-P. Nugroho, A. (2018) Generational diversity, overconfidence and decision-making in family business: a knowledge heterogeneity perspective. Asia Pacific Management Review, Vol. 23, p. 53-59.

Vicente, R. B. Lima, L. P. Ciribeli, J. P. Pierre, F. A. O. (2015) Análise bibliométrica de estudos sobre empresas familiares publicados nos Encontros da ENANPAD - Durante os anos de 1997 a 2012. XXVI ENANGRAD, Anais. Foz do Iguaçu, 25 a 27 de outubro.

Villagómez de Anderson, M. (2015) Gobierno de las empresas familiares y su importancia em la economía. Quito: UASB, 3 de septiembre de 2015. Ponencia. Datos del Instituto Ecuatoriano de Gobernanza Corporativa, 2015. 
Whetten, D. A. (2007) A critique of organizational identity scholarship: Challenging the uncritical use of social identity theory when social identities are also social actors. In: C. A. Bartel, S. L. Blader, A. Wrzesniewski, eds. Identity and the Modern Organization. Lawrence Erlbaum Associates, Mahwah, NJ, p. 253-272.

Wry, T. York, J. G. (2017) An identity-based approach to social Enterprise. Academy of Management Review, vol. 42, No. 3, p. 437-460. England: Cambridge University Press.

Gregory, S. A. (1966). The Design Method. Butterworths.

Harmon, M. M. (1989). The Simon/Waldo debate: A review and update. Public Administration Quarterly, 12(4), 437-451.n

Hassard, F. (2009). Culture, inheritance and identity: Towards an idemological perspective. Paper presented at the Symposium on Art and Science-Session I, InterSymp 2009: The 21st international conference on systems research, informatics and cybernetics, August 3-7, Baden-Baden, Germany.

James, S., \& Ben-gera, M. (2004). A comparative analysis of government offices in OECD countries (GOV/PGC/MPM/RD(2004)1). Paris, France: Organisation for Economic Co-operation and Development.

Kamprani, K. (2009). The uncomfortable. Retrieved March, 2015, from http://www.kkstudio.gr/projects/the-uncomfortable.

Kesler, G., \& Schuster, M. H. (2009). Design your governance model to make the matrix work. People \& Strategy, 32(4), 16-27.

Laurente, A. (1981). Matrix organizations and Latin cultures. International Studies of Management \& Organization, 10(4), 101-114.

Lawrence, P. R., Kolodny, H. F., \& Davis, S. M. (1977). The human side of the matrix. Organizational Dynamics, 6(1), 43-61.

Likert, R. (1976). Improving cost performance with cross-functional teams. Management Review, 65(3), 36

Ludwig, S. (1970). The move to matrix management. Management Review, 59(6), 60.

Marini, C., \& Martins, H. F. (2008). Matrix government: Networked structures to generate development results. Paper presented at the 8th international seminar on the theories of China's finance and economics and frontline practice, October, Beijing.

Martins, H. F. (2004). A theory of policy fragmentation. Brasília, Brazil: Publix Institute.

Merrian-webster's Collegiate Dictionary. (2015). Design. In Merriam Webster's collegiate dictionary. Retrieved March, 2015, from http://www.merriam- webster.com/dictionary/design.

Newell, A., \& Simon, H. (1972). Human problem solving. Englewood Cliffs, NJ: Prentice-Hall.

Oecd. (2003). Coerência nas políticas: Relatório final de atividades (March 2003). Paris, France: Comitê de Gestão Pública/Organisation for Economic Co-operation and Development.

Pahl, G., \& Beitz, W. (1996). Engineering design: A systematic approach (2nd ed.). London: Springer.

Pc Magazine (2012). Prototyping [definition of]. In Computer Language Company Inc., Encyclopedia. Retrieved May 3, 2012, apud http://en.wikipedia.org/wiki/Prototype.

Peters, B. G. (1998). Managing horizontal government: The politics of coordination (Research paper, no. 21). Ottawa, Canada: Canadian Centre for Management Development.

Ralph, P. (2010). Comparing two software design process theories. In R. Winter, J. L. Zhao, \& S. Aier (Eds.), Proceedings of the 5th international conference on Design Science Research in Information Systems and Technology (DESRIST 2010): "Global Perspectives on Design Science Research" (pp. 139-153). Berlin, Germany: Springer.

Simon, H. A. (1946). The proverbs of administration. Public Administration Review, 6(1), 53-67.

Simon, H. A. (1996). The sciences of the artificial. Cambridge, MA: MIT Press.

Sy, T., \& D'annunzio, L. S. (2005). Challenges and strategies of matrix organizations: Top-level and mid-level managers' perspectives. Human Resource Planning, 28(1), 39-48.

Tavis, A. (2013). Perspectives. People \& Strategy, 36(1), 4.

Trommel, W., Verschuere, B., \& Brandsen, T. (2014). Manufactured civil society: An emerging government strategy. ESADE Bulletin, May. Barcelona, Spain: Institute for Public Governance and 
Management, ESADE, Ramon Llull University.

Waldo, D. (1955). The study of public administration. Garden City, NY: Doubleday.

Wessal, A., Treuth, M., \& Wescott, C. (2013). Science of delivery: Implications for monitoring and evaluation. Mimeo, available at http://dx.doi.org/10.2139/ssrn.2302390.

World Bank. (2010). Limitations. GET note: Center of government delivery units "Recently asked questions" series. Global Expert Team. Retrieved March, 2015, from http://siteresources.worldbank.org/EXTGOVANTICORR/Resources/3035863-

1285601351606/NovemberGetNote.pdf. 